\title{
Predictive Quantization for Data Volume Reduction in Staggered SAR Systems
}

\author{
Michele Martone, Nicola Gollin, Michelangelo Villano, Member, IEEE, \\ Paola Rizzoli, and Gerhard Krieger, Fellow, IEEE,
}

\begin{abstract}
Staggered synthetic aperture radar (SAR) is an innovative SAR acquisition concept which exploits digital beamforming (DBF) in elevation to form multiple receive beams and continuous variation of the pulse repetition interval to achieve high-resolution imaging of a wide continuous swath. Staggered SAR requires an azimuth oversampling higher than a SAR with constant PRI, which results in an increased volume of data. In this paper, we investigate the use of linear predictive coding, which exploits the correlation properties exhibited by the non-uniform azimuth raw data stream. According to this, a prediction of each sample is calculated on board as a linear combination of a set of previous samples. The resulting prediction error is then quantized and downlinked (instead of the original value), which allows for a reduction of the signal entropy and, in turn, of the onboard data rate achievable for a given target performance. In addition, the a-priori knowledge of the gap positions can be exploited to dynamically adapt the bit rate allocation and the prediction order to further improve the performance. Simulations of the proposed Dynamic Predictive Block-Adaptive Quantization (DP-BAQ) are carried out considering a Tandem-L-like staggered SAR system for different orders of prediction and target scenarios, demonstrating that a significant data reduction can be achieved with a modest increase of the system complexity.
\end{abstract}

Index Terms-Staggered synthetic aperture radar, block adaptive quantization (BAQ), data reduction, linear predictive coding.

\section{INTRODUCTION}

$\mathbf{S}$ YNTHETIC aperture radar (SAR) represents nowadays a well-recognized technique for a broad variety of remote sensing applications, being able to acquire high-resolution images of the Earth's surface independently of sunlight and weather conditions. However, conventional SAR is constrained by the pulse repetition frequency (PRF) for the imaging of wide swaths and, at the same time, of fine azimuth resolutions. To overcome these limitations, in the last decades innovative spaceborne radar techniques have been proposed, which allow for high-resolution imaging of a wide swath width by exploiting multiple azimuth channels (MAC) and digital beamforming (DBF) in elevation to achieve Scan-on-Receive (SCORE) [1], [2], [3], [4], [5], [6]. Alternatively, single-channel SAR based on the simultaneous recording of multiple echo pulses received from different elevation directions (so-called "multibeam" mode) [7], [8] enables a further increase of the imaged area by keeping the antenna length within reasonable limits and avoiding the

The authors are with the Microwaves and Radar Institute, German Aerospace Center (DLR), 82234 Wessling, Germany. e-mail: Michele.Martone@dlr.de employment of burst modes. Such systems are still limited by the presence of blind ranges across the swath, which arise since the radar cannot receive while transmitting. The opportunity of exploiting the variation of the PRI to solve the blind range problem was first proposed independently in [9] and in [7], [10]. Then, the idea culminated in the staggered SAR concept, which includes a refined design of the PRI sequences, the use of proper interpolation on the raw data and consideration of the ambiguities [11], [12], [13], [14]. By cyclically changing the pulse repetition interval (PRI), Staggered SAR allows to vary (i.e., to "stagger") the range positions of such data gaps along the azimuth dimension. In this way, high-resolution imaging of a large continuous swath of up to $350 \mathrm{~km}$ without the need for a long antenna with multiple apertures becomes possible [11].

The requirement of swath width and resolution, together with the use of large bandwidths and multiple acquisition channels, is clearly associated to the generation of a large volume of data, which implies, from the mission design point of view, harder requirements in terms of onboard memory and downlink capacity. In the context of single-channel staggered SAR systems, a method consisting of an interpolation combined with low-pass Doppler filtering and decimation of the acquired raw data has been proposed in [13], [15], which allows for a data reduction of up to $50 \%$ at the cost of a significant onboard computational effort. In this scenario, SAR raw data quantization represents an aspect of utmost importance, since the number of bits employed to digitize the recorded radar signal, on the one hand, directly affects the performance of the resulting SAR products and, on the other hand, defines the total amount of data to be managed by the system.

Nowadays, one of the most widely used methods for SAR raw data compression is the block-adaptive quantization (BAQ). BAQ is a lossy data reduction technique which employs a space-varying estimation of the raw data statistics in order to set the quantization decision levels [16], [17]. BAQ offers a good trade off between scheme complexity (a simple scalar quantizer), achievable compression ratio, and resulting image quality, and represents therefore an attractive solution for data volume reduction in spaceborne SAR systems. In the last years, novel compression algorithms have been proposed to allow for a finer performance and resource optimization, based on the implementation of non-integer quantization rates [18], combined with data-driven compression schemes [19], [20], [21]. Further, possible strategies for data volume reduction in the context of multi-channel SAR systems have 
been proposed in [22], [23].

Conventional SAR raw data usually show very little correlation among nearby samples, which can be only partially used for compression algorithms. On the other hand, in staggered SAR a significant azimuth oversampling is mandatory to properly reconstruct the information lost within the blind ranges [13]. The resulting data redundancy can be therefore exploited to reduce the volume of data to be acquired and stored on board.

This paper addresses the use of linear predictive coding for onboard data reduction in staggered SAR systems. Linear predictive coding [24] exploits the existing correlation between adjacent azimuth samples, i.e., samples which are located at successive range lines and within the same range bin. Such a correlation is introduced by the antenna pattern and the azimuth oversampling: a prediction of each sample is estimated on board through a linear combination of a set of previously received samples in the azimuth dimension. The difference between the sample and its prediction is then quantized on board and downlinked. The resulting prediction error is characterized by a signal entropy which is smaller than the one of the original SAR raw data. This allows for an efficient encoding of the data stream and, in turn, for a reduction of the number of quantization bits for a given target performance, at the cost of a modest computational burden. Moreover, the proposed method preserves the nonuniform sampling of the staggered SAR data, making possible to employ more advanced processing techniques on ground, which would be more difficult to apply to resampled raw data as proposed, e.g., in [25]. The use of predictive quantization in the context of conventional SAR has been previously investigated in [26], [27].

The paper is organized as follows. The proposed method for data volume reduction based on linear predictive quantization, its detailed mathematical formulation, and application for onboard data volume reduction in staggered SAR are discussed in Section II. Moreover, a strategy to effectively reconstruct the blind ranges, through a dynamic selection of the prediction order and of the bit allocation in the vicinity of the gap positions, followed by a proper data interpolation, is proposed as well. Simulations for different orders of prediction and target scenarios are presented in Section III for a Tandem-L-like staggered SAR system and demonstrate the effectiveness of the proposed compression scheme. Finally, Section IV concludes the paper.

\section{Dynamic PREDictive Block-Adaptive QUANTIZATION FOR STAGGERED SAR SYSTEMS}

\section{A. SAR Signal Statistical Characterization}

It is well known that the In-phase (I) and Quadrature (Q) components of the SAR raw signal can be described as zero-mean Gaussian stationary and independent processes with a slowly changing variance in both range and azimuth directions [28]. This assumption holds as a consequence of the central limit theorem (CLT) and is almost independent of the type of spaceborne SAR sensor used (frequency, resolution) as well as of the characteristics of the scene under illumination: indeed, a very large number of targets overlap their response in the raw data domain within the imaged scene. This is due, in turn, to the large extension on ground of the azimuth antenna footprint and of the range pulse, which, for the considered spaceborne SAR systems, are in the order of several kilometers. The samples of the SAR raw azimuth signal $s(t)$, received at different time instants, can be modeled as partially correlated random variables. Such a correlation is introduced by the azimuth antenna pattern (or Doppler spectrum) and by the selected pulse repetition frequency (PRF), and can be described by the normalized autocorrelation function $R_{s}(\tau)$ as

$$
R_{s}(\tau)=\mathbb{E}\left\{s^{*}(t) \cdot s(t+\tau)\right\} / \mathbb{E}\left\{|s(t)|^{2}\right\},
$$

where $\tau$ represents the time lag in the azimuth dimension. Correlation in the range dimension is not considered here due to the negligible data oversampling (indeed, the range bandwidth is typically sharply limited and only a small oversampling is usually employed). The azimuth autocorrelation function can be expressed as the inverse Fourier transform of the Doppler power spectral density $P_{u}(f)$

$$
R_{s}(\tau)=\mathcal{F}^{-1}\left\{P_{u}(f)\right\},
$$

where $f$ is the Doppler frequency. If a uniformly illuminated rectangular azimuth aperture of length $L$ is considered, the power spectral density can be expressed as [29], [30]

$$
P_{u}(f)=\sin ^{4}\left(\pi \frac{L}{2 v_{s}} f\right) /\left(\pi \frac{L}{2 v_{s}} f\right)^{4},
$$

being $v_{s}$ the satellite velocity. The above equation allows for the derivation of the autocorrelation in closed form as [29]

$$
R_{s}(\tau)= \begin{cases}\frac{3}{4}\left(B_{R} \cdot \tau\right)^{3}-\frac{3}{2}\left(B_{R} \cdot \tau\right)^{2}+1 & 0 \leq \tau \leq \frac{1}{B_{R}} \\ -\frac{1}{4}\left(B_{R} \cdot \tau-2\right)^{3} & \frac{1}{B_{R}} \leq \tau \leq \frac{2}{B_{R}} \\ 0 & \text { elsewhere. }\end{cases}
$$

In the above equation $B_{R}$ represents the bandwidth of the spectral power density function and is defined as

$$
B_{R}=\frac{2 v_{s}}{L} \text {. }
$$

Hence, longer antennas give a more directive beam, which can be considered as a narrower low-pass filter in the Doppler domain. On the other hand, a lower satellite velocity results in a higher correlation time, since, for a given time lag $\tau$, two targets will be more overlapped in the raw data space and therefore more similar to each other.

It is worth pointing out that the function in (4) represents the correlation which is introduced by the system for a white noise input. Indeed, there could be additional correlation due to the properties of the scene under illumination (e.g., point-like targets). Hence, using only the system-induced correlation represents also a sort of worst case scenario, i.e. fully developed speckle.

As already mentioned, for staggered SAR systems a certain azimuth oversampling is necessary to properly recover the raw data information in the neighborhood of gaps introduced 
during the SAR acquisition. The azimuth oversampling factor $o_{f}$ is defined as the ratio between the pulse repetition frequency and the processed Doppler bandwidth, of $=$ $\mathrm{PRF} / \mathrm{PBW}$. In this scenario, we propose a compression algorithm based on differential pulse code modulation (DPCM), which aims at exploiting the correlation exhibited by adjacent azimuth samples by encoding, instead of the original raw data sample, the difference between the original one and its prediction. This allows for a reduction of the signal dynamic (i.e., its entropy), and hence for a decrease of the required bit rate for a given quantization performance [24]. By having a-priori information on the raw signal statistics, a proper design of the predictor can be implemented, as it is detailed in the following.

\section{B. Mathematical Formulation and Implementation}

Let $s[\mathrm{n}]$ be the raw azimuth sample taken at the discrete time instant $\mathrm{n}$. According to the linear prediction theory, the estimate of the sample $s[\mathrm{n}], \tilde{s}[\mathrm{n}]$, is a linear combination of its $N_{p}$ preceding samples

$$
\tilde{s}[\mathrm{n}]=\sum_{k=1}^{N_{p}} \beta_{k} s[\mathrm{n}-\mathrm{k}],
$$

where $N_{p}$ defines the so-called prediction order, while $\beta_{k}$ is the weight associated to the $k$-th previous sample $s[\mathrm{n}-\mathrm{k}]$. From this, we define the prediction error $d[\mathrm{n}]$ as

$$
d[\mathrm{n}]=s[\mathrm{n}]-\tilde{s}[\mathrm{n}] .
$$

The set of weights $\boldsymbol{\beta}=\left\{\beta_{1}, \beta_{2}, \ldots, \beta_{N_{p}}\right\}$ is chosen to minimize the mean square prediction error and is derived as [24]

$$
\beta=C^{-1} \rho,
$$

where $C \in \mathbb{R}^{N_{p} \times N_{p}}$ is the covariance matrix of the random process $s[\mathrm{n}]$ and is populated by the correlation values among the $N_{p}$ preceding samples used for the prediction, i.e., considering two samples at discrete time instants $i$ and $j$,

$$
C_{i, j}=R_{s}[|i-j| \cdot \mathrm{PRI}] .
$$

Furthermore, $\boldsymbol{\rho} \in \mathbb{R}^{N_{p}}$ represents the vector of the correlation values between the $N_{p}$ previous samples and the sample to be predicted at the time instant $n$, i.e.,

$$
\rho_{k}=R_{s}[k \cdot \mathrm{PRI}] .
$$

The encoding process is shown in Fig. 1: the prediction error $d[\mathrm{n}]$, derived as in (7), is given as input to the block-adaptive quantizer (block "BAQ"). The quantized prediction error $d_{q}[\mathrm{n}]$ is the information which is actually downlinked to the ground, but it is also used on board in a feedback loop, together with the sample prediction $\tilde{s}[\mathrm{n}]$, in order to obtain a quantized version of the true input signal $\hat{s}[\mathrm{n}]$ as

$$
\hat{s}[\mathrm{n}]=\tilde{s}[\mathrm{n}]+d_{q}[\mathrm{n}] .
$$

This quantity, in turn, is then used as input for the prediction of the next sample. The decoding process is shown in Fig. 2: the received signal is first decoded (block "BAQ ${ }^{-1}$ ") and then the same prediction loop is implemented to finally get

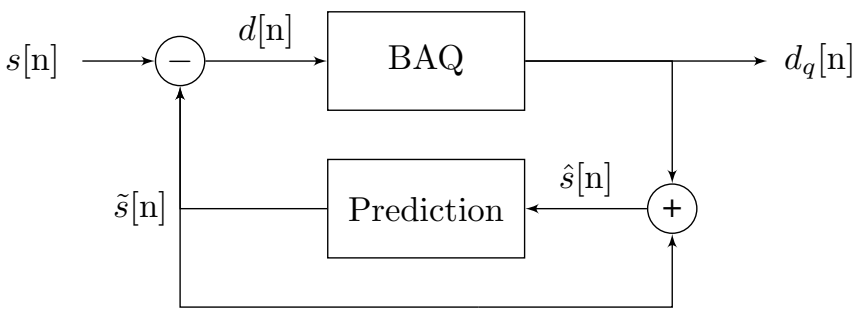

Fig. 1. Predictive quantization encoding flow scheme.

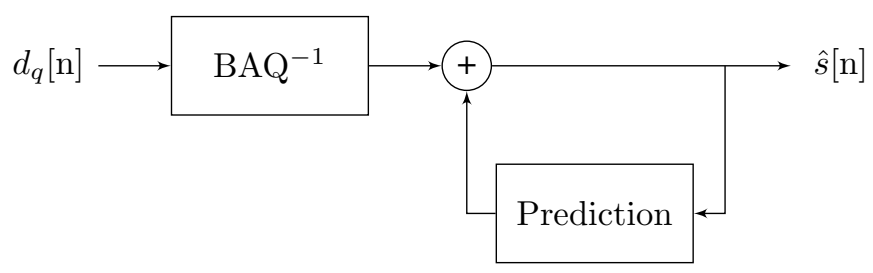

Fig. 2. Predictive quantization decoding flow scheme.

a quantized version of the original SAR raw data sample $\hat{s}[\mathrm{n}]$ as in (11). It is worth to point out that the prediction block takes as input the quantized version of the prediction error, indeed $\hat{s}[\mathrm{n}]$. In this way, the exact same sample value $d_{q}[\mathrm{n}]$ is employed both, at encoding and decoding stage, hence avoiding stability problems due to the propagation and accumulation of reconstruction errors in the feedback loop. The scheme depicted in Fig. 1 and Fig. 2 refers to a prediction order $N_{p}=1$. Obviously, if $N_{p}>1$ a corresponding number of preceding samples needs to be used for prediction in the feedback loop as in (6).

It is worth to point out that, for the investigations conducted in this paper, a causal predictor has been considered, i.e., only preceding samples are used in the prediction process, according to (6). A causal filter represents the simplest prediction scheme, which minimizes the required onboard storage and computational effort, if compared to other predictor types and, e.g., the alternative method proposed in [15]. For a causal predictor, the decoder reconstructs each sample by using the information (i.e., the quantized prediction errors) received at previous time instants, and both the encoding and decoding loops are implemented by a recursive filter, as shown in Fig. 1 and Fig. 2. Equivalently, an anti-causal filter could be also employed: in this case, the encoder cannot operate in real time (since it needs first to "wait" for the future samples needed for the prediction), hence requiring an increased onboard complexity for its implementation. Then, the decoder reconstructs the data stream from the last received sample to the first one (i.e., "in the opposite direction" with respect to a causal prediction scheme). A causal predictor achieves the same performance gain of an anti-causal one of the same order, being the autocorrelation of the SAR raw signal an even function, and both require, for their implementation, the storage in the onboard memory of at least of two range lines at a time, i.e., one for the prediction and the actual one for calculating the prediction error. 
Different is the scenario represented by a non-causal filter, where both past and future samples are exploited in the prediction process, and at least three range lines need to be stored at a time (i.e., two for the prediction and the actual one for calculating the prediction error). Similarly to an anti-causal predictor, a non-causal filter must wait for future sample(s) to derive the corresponding prediction value, hence requiring an increased implementation complexity. For this, a relevant issue is represented by the fact that, if for a causal predictor the samples used for the prediction are, in their turn, the result of a prediction and quantization operation within the recursive loop (as in Fig. 1), this is not true for a non-causal predictor: here, the future samples employed in the prediction filter are used "as is", whereas, at the decoding stage, only the quantized version of the prediction errors is available. This inconsistency between the information available at the encoding stage and the one available at decoding stage must be carefully taken into account in order to avoid stability problems in the reconstruction loop due to possible error propagation caused by quantization. Moreover, for a non-causal predictor the reconstruction process cannot be carried out "sample by sample" (differently from a causal or an anti-causal filter), but must be approached as a linear equations system, where each equation represents the linear combination of the preceding/following samples (this operation requires additional processing effort on ground, which, however, does not represent a critical aspect). To conclude, non-causal prediction can in principle be used for data volume reduction in a staggered SAR system. However, the possible performance improvement with respect to a causal or an anti-causal predictor, which is implied by the better exploitation of the correlation between neighboring samples, must be traded by taking into account the increased scheme complexity and the larger number of samples to be stored on board, and by considering the impact of all the aforementioned aspects. These will be addressed in details in future research studies and investigations.

The performance gain $G_{N_{p}}$ obtained with a $N_{p}^{\text {th }}$-order predictor is expressed as the ratio between the variance of the prediction error $\sigma_{\mathcal{D}, N_{p}}^{2}$ and the one of the input signal $\sigma_{\mathcal{S}}^{2}[24],[31]$

$$
G_{N_{p}}=\frac{\sigma_{\mathcal{D}, N_{p}}^{2}}{\sigma_{\mathcal{S}}^{2}},
$$

being $\mathcal{S}$ and $\mathcal{D}$ the random variables representing the sample to be estimated and the prediction error (estimated according to (7)), respectively. That is, the smaller the dynamic of the prediction error, the larger the resulting coding gain.

Since the prediction error is obtained as a linear combination of $N_{p}$ Gaussian random variables (see Section II-A), according to (6) and (7), $d[\mathrm{n}]$ is also Gaussian (this is strictly true if no quantization is applied in the recursive loop, but holds in practice with reasonable accuracy). The Gaussian nature of the prediction error implies that a reduction of the signal dynamic, achieved by means of the prediction process, results in a decrease of its information entropy. For a given set of system parameters, this allows for the derivation, in closed form, of the prediction gain $G_{N_{p}}$, by estimating the standard deviation of the prediction error $\sigma_{\mathcal{D}, N_{p}}$ and substituting it into (12). Let us consider the simplest case of a $1^{\text {st }}$-order predictor, where $N_{p}=1$ and the previous sample only is used in the prediction filter. According to the notation used in (7), the random variable $\mathcal{D}$ is obtained as the difference between the random variables $\mathcal{S}$ and $\tilde{\mathcal{S}}$ as

$$
\mathcal{D}=\mathcal{S}-\tilde{\mathcal{S}} \sim \mathcal{N}\left(0, \sigma_{\mathcal{S}}^{2}+\sigma_{\tilde{\mathcal{S}}}^{2}-2 \rho_{1} \cdot \sigma_{\mathcal{S}} \sigma_{\tilde{\mathcal{S}}}\right)
$$

being $\rho_{1}$ the autocorrelation value obtained for the time lag $\tau=$ PRI, according to (10), where, in turn, $R_{s}(\tau)$ is expressed in (4). By modeling the input signal as a stationary random process (i.e., its unconditional probability distribution does not change in time), due to the weighting introduced in the prediction process, it follows that

$$
\sigma_{\tilde{\mathcal{S}}}=\beta_{1} \sigma_{\mathcal{S}}
$$

According to (8), $\beta_{1}=\rho_{1}$ (being $C_{1,1}=1$, see (9)) and the variance of the prediction error in $(13), \sigma_{\mathcal{D}, N_{p}=1}^{2}$, can be further simplified as

$$
\sigma_{\mathcal{D}, N_{p}=1}^{2}=\sigma_{\mathcal{S}}^{2}\left(1-\rho_{1}^{2}\right) .
$$

By substituting (15) in (12), the prediction gain for a $1^{\text {st }}$-order predictor can be finally expressed as

$$
G_{1}=\left(1-\rho_{1}^{2}\right)^{-1} \text {. }
$$

The prediction gains $G_{N_{p}}$ for $N_{p}=\{2,3,4\}$ are derived by following the same procedure and are expressed as

$$
\begin{aligned}
G_{2}= & \left(1+\beta_{1}^{2}+\beta_{2}^{2}+2 \rho_{1}\left(\beta_{1} \beta_{2}-\beta_{1}\right)-2 \rho_{2} \beta_{2}\right)^{-1}, \\
G_{3}= & {\left[1+\beta_{1}^{2}+\beta_{2}^{2}+\beta_{3}^{2}+\right.} \\
& +2 \rho_{1}\left(\beta_{1} \beta_{2}+\beta_{2} \beta_{3}-\beta_{1}\right)+ \\
& \left.+2 \rho_{2}\left(\beta_{1} \beta_{3}-\beta_{2}\right)-2 \rho_{3} \beta_{3}\right]^{-1}, \\
G_{4}= & {\left[1+\beta_{1}^{2}+\beta_{2}^{2}+\beta_{3}^{2}+\beta_{4}^{2}+\right.} \\
& +2 \rho_{1}\left(\beta_{1} \beta_{2}+\beta_{2} \beta_{3}+\beta_{3} \beta_{4}-\beta_{1}\right)+ \\
& +2 \rho_{2}\left(\beta_{1} \beta_{3}+\beta_{2} \beta_{4}-\beta_{2}\right)+ \\
& \left.+2 \rho_{3}\left(\beta_{1} \beta_{4}-\beta_{3}\right)-2 \rho_{4} \beta_{4}\right]^{-1} .
\end{aligned}
$$

The weights $\left\{\beta_{n}\right\}$ are obtained from (8) for each prediction order. Their expression as function of the autocorrelation values $\left\{\rho_{n}\right\}$ is not included in the above equations to avoid large formulations.

For data digitization, a block-adaptive quantizer (BAQ) is considered, which, as it has been already mentioned, exploits the input signal statistics to perform a block-wise quantization of the SAR raw data [16]. Hence, the encoding/decoding schemes pictured in Fig. 1 and Fig. 2 are implemented on samples located in successive range lines and for blocks of $N_{\text {Block }}$ range samples: first, the prediction process is carried out for each of the $N_{\text {Block }}$ range samples (i.e., located at $N_{\text {Block }}$ consecutive range bins) independently; then, once the difference from the data block and its prediction has been calculated, azimuth sample by azimuth sample for each range bin, the BAQ adapts the quantization levels to the statistics of the corresponding prediction error block. The 


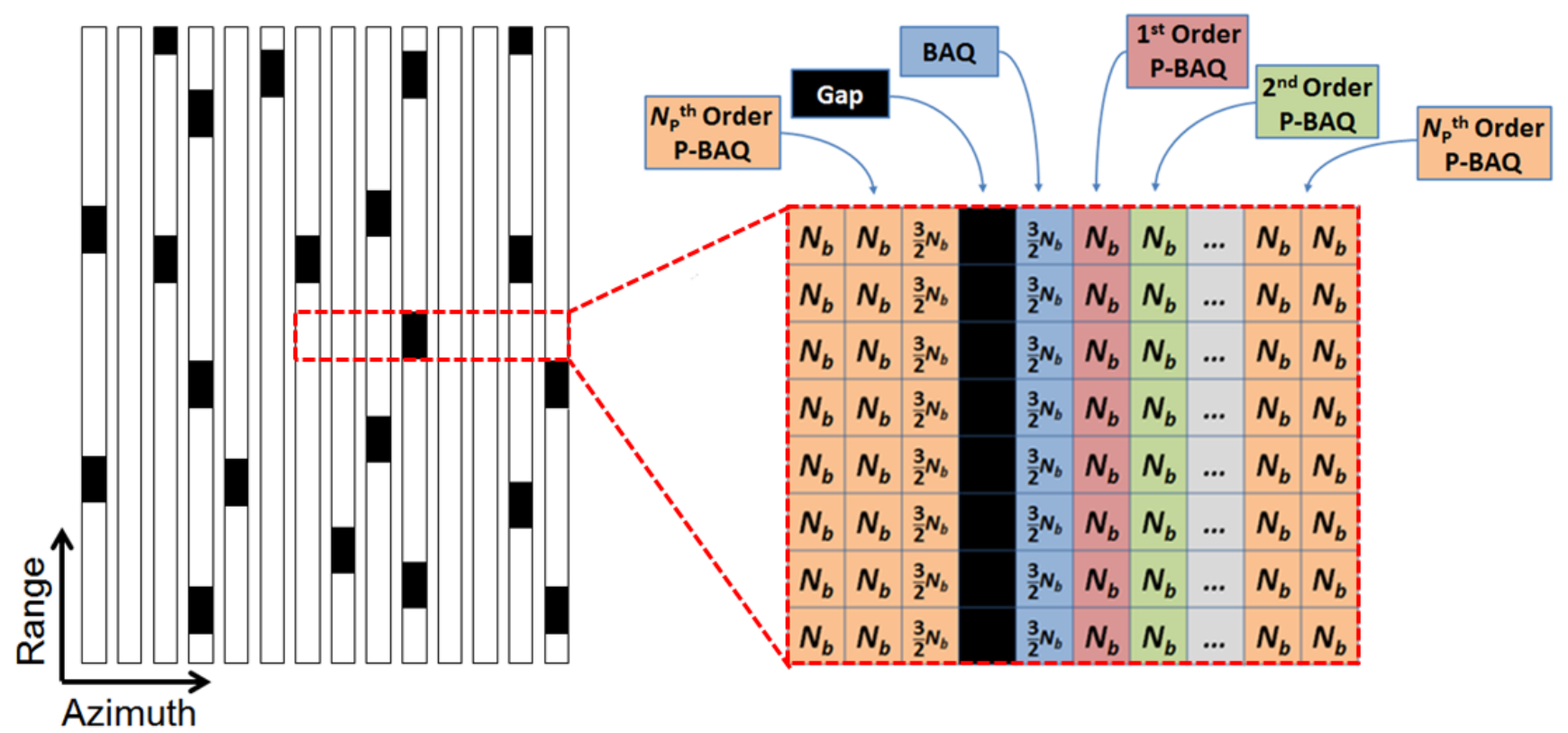

Fig. 3. (Left) Distribution of the blind ranges (black rectangles) within a staggered SAR acquisition. Each gap region typically extends by several hundreds of samples in the range direction. (Right) Zoom-in of a raw data region affected by gaps. Each cell corresponds to a raw data sample. The proposed method is implemented by jointly applying a variable bit rate allocation (indicated in each box, where $N_{b}$ represents the mean bit rate in bits/sample) together with a dynamic selection of the prediction order (depicted with different colors and shown on the top of the figure) in the gap vicinity.

BAQ is optimized for Gaussian signals (which is the case of the prediction error), and a Cartesian scheme is usually implemented, i.e., the I and Q components of the complex raw signal are separately treated and quantized.

\section{Gap mitigation}

The location of blind ranges (gaps) along the azimuth dimension of a staggered SAR acquisition and their range extension is related to the specific sequence of PRI employed, and is therefore known a priori (i.e. at commanding time). This valuable information can be exploited to dynamically optimize the bit rate allocation and to adapt the prediction process for the samples located in the gap vicinity, in order to better recover the missing information and, ultimately, to improve the overall signal reconstruction quality. The left-hand side of Fig. 3 shows a hypothetical distribution of the blind ranges (black rectangles) within a staggered SAR acquisition. The range and azimuth dimensions are indicated at the bottom-left of the figure. Each vertical stripe represents a single range line, while each gap region ideally extends by many hundreds of samples along the range dimension. In particular, if elaborated non-uniform PRI sequences are employed, gaps can be displaced in range such that two consecutive azimuth samples at the same range are never missed [13]. The right-hand side of Fig. 3 shows a zoom of the raw data matrix which is affected by a blind range (in black). Each cell corresponds to a raw data sample. The proposed technique is implemented by jointly applying a variable bit rate allocation (which is provided in each box; $N_{b}$ represents the mean bit rate in bits/sample) together with a dynamic selection of the prediction order in the gap vicinity. In particular, the order of the prediction filter is dynamically set as indicated on the right-hand side of Fig. 3. The first azimuth sample after the gap is quantized by means of a standard BAQ (i.e. no prediction is applied). Then, the following sample (in red) is encoded through a $1^{\text {st }}$-order predictor (P-BAQ), which exploits the information carried out by the previous sample only. The next one (in green) is then encoded through a $2^{\text {nd }}$-order predictor (in general, the $m$-th sample after the gap is encoded with a predictor filter of order $m-1$ ). The operation is repeated until the operative prediction order $N_{o p}$ is reached, where $N_{o p}$ is defined at system design stage as trade off between system complexity and achievable prediction gain (this relevant aspect will be further detailed in the next section). The described precaution, which consists of an adaptive selection of the order of the prediction filter (as sketched by the different colors on the right-hand side of Fig. 3), aims at excluding the missing samples in the prediction process, hence allowing for a better reconstruction of the signal in the immediate gap vicinity and, in turn, of the missing sample itself after proper BLU interpolation. Indeed, the prediction filter is designed under the assumption that the SAR raw data can be modeled as a stationary random process (i.e. their unconditional probability distribution does not change in time), and therefore the gaps are in principle not taken into account in the weight definition in (8). If, on the other hand, a constant $N_{p}^{\text {th }}$-order predictor were used, this would result in a larger prediction error, hence degrading the performance gain in (12).

Possible non-stationarities may be caused by temporal changes of the target during the integration time, which, for typical spaceborne SAR systems, are in the order of a few seconds. Such a temporal decorrelation leads to an increase of the Doppler bandwidth and, in turn, to a worsening of the performance (e.g., defocusing) in the resulting SAR image. 
TABLE I

BIT ALLOCATION APPLIED TO THE SAMPLES IN THE GAP VICINITY.

\begin{tabular}{|c|c|c|c|c|c|}
\hline bit rate & $\begin{array}{c}\text { before } \\
\text { gap }\end{array}$ & on gap & $\begin{array}{c}\mathbf{1}^{\text {st }} \text { after } \\
\text { gap }\end{array}$ & $\begin{array}{c}\mathbf{2}^{\text {nd }} \text { after } \\
\text { gap }\end{array}$ & $\begin{array}{c}\mathbf{3}^{\text {rd }} \text { after } \\
\text { gap }\end{array}$ \\
\hline 2 & 3 & 0 & 3 & 2 & 2 \\
\hline 3 & 4 & 0 & 4 & 4 & 3 \\
\hline 4 & 6 & 0 & 6 & 4 & 4 \\
\hline 6 & 8 & 0 & 8 & 8 & 6 \\
\hline
\end{tabular}

However, in the present scenario, azimuth samples which are only a few PRI intervals apart are used in the prediction filter. Even for a very long predictor of, e.g., $10^{\text {th }}$ order $\left(N_{p}=10\right)$, a maximum time lag $\Delta t=N_{p} \cdot \mathrm{PRI} \approx 3.7 \mathrm{~ms}$ is obtained, which is much shorter than the decorrelation time typically observed for any kind of vegetation imaged at $\mathrm{X}$ band at moderate to high wind speeds, as discussed in [32].

Regarding the variable bit rate allocation to be applied in the gap vicinity, it is worth noting that the bits originally used for a missing sample could be ideally not downloaded at all, i.e. 0 bits are used. Indeed, we found that the best way to exploit the gained $N_{b}$ bits is, as expected, to allocate them, in equal measure, before and after the gap (i.e., a "distributed" bit allocation), leading to the $N_{b}+\frac{1}{2} N_{b}=\frac{3}{2} N_{b}$ bits/sample for the samples in the gap vicinity on the right-hand side of Fig. 3. Since the BAQ operates at certain integer bit rates, the actual bit allocation in the neighborhood of a gap is defined in Table I for bit rates of 2, 3, 4, and 6 bits/sample. These are indicated in the first column of the table ("bit rate") and each of them represents the average of the bit rate values in the corresponding table row.

To obtain focused images, staggered SAR raw data need to be first interpolated on a uniform grid. For this purpose, a Best Linear Unbiased (BLU) interpolation is employed [11], [15], which exploits the correlation between neighbouring azimuth samples to optimally estimate the values on the output grid and to reconstruct the samples in correspondence of a gap. This efficient allocation allows for a consistent mitigation of the errors introduced by the combined effect of quantization and interpolation. Indeed, we have verified that this simple but effective strategy allows for a quality of the reconstructed signal (in terms of error power) which is practically equivalent to the one obtained for gap-free data.

The proposed method jointly exploits a dynamic bit rate allocation and a variable prediction order in the gap vicinity and is therefore named Dynamic Predictive Block-Adaptive Quantization (DP-BAQ), whose effectiveness for data volume reduction is demonstrated in the next section for a real mission scenario.

\section{Simulation Results}

Staggered SAR is currently considered as the baseline acquisition mode for Tandem-L, a German Aerospace Center (DLR) proposal for a highly innovative L-band single-pass interferometric and fully polarimetric radar satellite mission to monitor dynamic processes on the Earth surface [33]. A list of the system parameters for the Tandem-L mission is given in Table II. In order to assess the proposed method for
TABLE II

TANDEM-L SYSTEM PARAMETERS.

\begin{tabular}{|l|l|}
\hline Parameter & Value \\
\hline Orbit height & $745 \mathrm{~km}$ (@ equator) \\
\hline Carrier frequency, $f_{c}$ & $1.25 \mathrm{GHz}(\mathrm{L}$ band $)$ \\
\hline Horizontal baselines & $800 \mathrm{~m} \ldots 20 \mathrm{~km}$ \\
\hline Revisit time & 16 days \\
\hline Range bandwidth & up to $84 \mathrm{MHz}$ \\
\hline Mean (staggered) PRF & $2700 \mathrm{~Hz}$ \\
\hline Doppler bandwidth, PBW & $1130 \mathrm{~Hz}$ \\
\hline Azimuth resolution & $7 \mathrm{~m}$ \\
\hline Swath width & $175 \mathrm{~km}($ quad) ...350 km (single/dual) \\
\hline Raw data quantization & BAQ @ 4 bits/sample \\
\hline Downlink capacity & $\sim 8 \mathrm{Terabyte} /$ day \\
\hline Reflector diameter & $15 \mathrm{~m}$ \\
\hline Mission lifetime & 10 years \\
\hline Polarization & single/dual/quad \\
\hline
\end{tabular}

data volume reduction, we carried out simulations of SAR raw data for a Tandem-L-like system in single-pol staggered SAR mode, and we compared the performance of different data compression algorithms. The steps followed for the present investigations are depicted in Fig. 4: SAR raw data are obtained by inverse focusing of simulated backscatter scenes. Then, standard BAQ as well as predictive quantization with constant (P-BAQ) and dynamic (DP-BAQ) selection for the bit rate and prediction order in the gap vicinity are applied for different bit rates to obtain a distorted version of the original data. The resulting non-uniform and quantized raw data are resampled to a uniform grid using a Best Linear Unbiased (BLU) interpolation and, finally, SAR focusing is performed. In this paper, the performance assessment for the different compression methods is conducted mainly on focused images, but an evaluation on SAR raw data is presented as well.

The azimuth pattern of the Tandem-L reflector antenna, with a diameter of $15 \mathrm{~m}$, can be well approximated by the one generated by a planar array with uniform aperture and azimuth length $L=10 \mathrm{~m}$ [11]. For the analyses presented in this paper, we considered the planar approximation since it allows for the expression of the theoretical autocorrelation function in closed form as in [30], and which is shown in Fig. 5 as a function of the time lag, $R_{s}(\tau)$ (in red). For Tandem- $\mathrm{L}$, the mean PRI is about $0.37 \mathrm{~ms}$, hence leading to a correlation between adjacent azimuth samples of about 0.67, which is identified by the dashed black lines in the figure. As introduced in Section I and summarized in Table II, such a high correlation is caused by the large system oversampling $o_{f}=\mathrm{PRF} / \mathrm{PBW} \sim 2.39$ (i.e., the data volume to be downlinked increases by almost $140 \%$ ), which is required for the proper staggered SAR operation.

Fig. 6 shows the theoretical gain as a function of the PRF up to the $6^{\text {th }}$-order predictor, derived as in (16)-(19). For this, a constant (i.e., not staggered) PRF has been employed. As expected, the larger the PRF, the larger the correlation among the raw data samples, the higher the resulting prediction gain. In particular, the upper boundary of the prediction gain strictly depends on the system characteristics (i.e., antenna size and shape of the azimuth antenna pattern, satellite velocity, and PRF, according to (3)-(5)), which directly affect the values of the correlation array $\rho$ and of the covariance matrix $C$. These, in turn, define the resulting gains according 


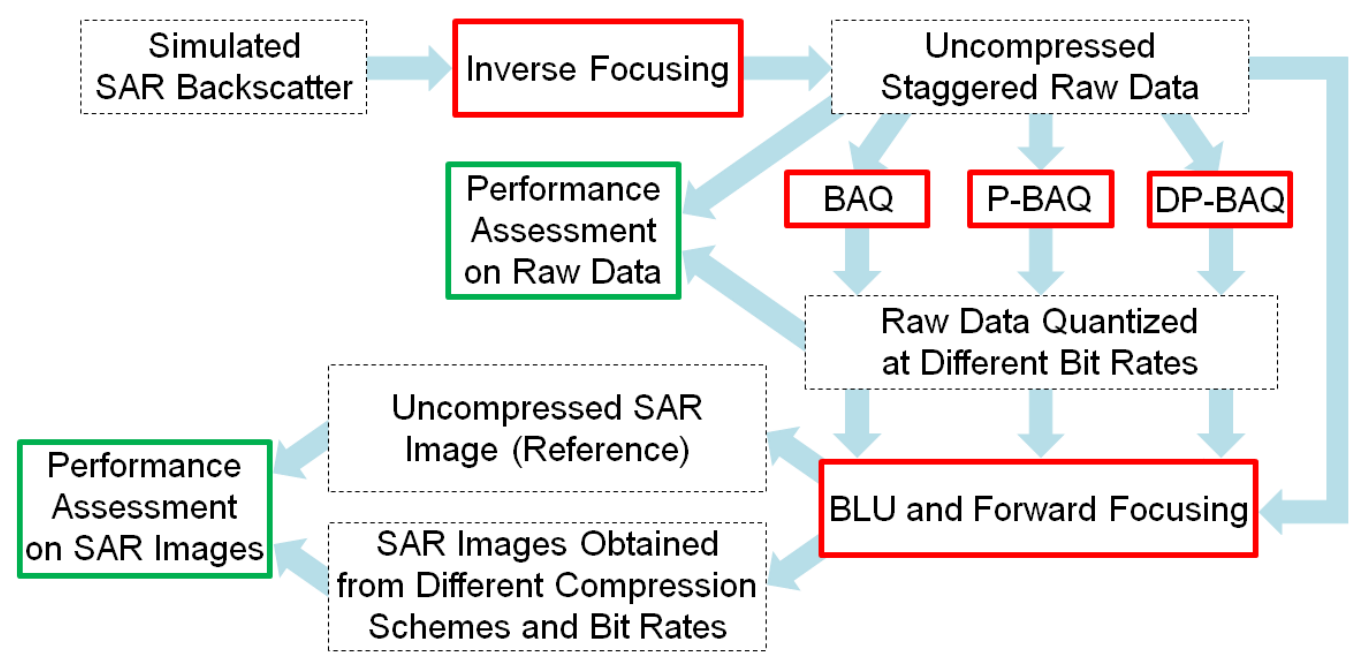

Fig. 4. Workflow for staggered SAR data simulation, quantization, and processing. The raw data are compressed using different compression schemes: a standard BAQ and a predictive quantizer with constant (P-BAQ) and dynamic (DP-BAQ) selection for the bit rate and prediction order in the gap vicinity. The resulting non-uniform and quantized raw data are resampled to a uniform grid using a Best Linear Unbiased (BLU) interpolation and, finally, SAR focusing is performed. The performance is evaluated on both, the raw data and the focused SAR images.

to the mathematical expression in (8) and (16)-(19). The best achievable prediction gain can therefore be numerically estimated once the specific system parameters are set (for a given PRF, the gains practically saturate beyond a certain prediction order). The oscillating behavior shown by the higher order predictors is due to the inversion of the covariance matrix $\boldsymbol{C}$ for the weights derivation in (8), in presence of very low correlation values: indeed, an $N_{p}$-order prediction gain $G_{N_{p}}$ starts to increase again when a not negligible correlation value of the same order (i.e., $\rho_{N_{p}}$ derived as in (10)) is obtained for the corresponding PRF value. The mean PRF of the single polarization mode of Tandem-L is $2700 \mathrm{~Hz}$ and is indicated by the dashed black line. For this, the prediction gain ranges between $2.5 \mathrm{~dB}$ and $5 \mathrm{~dB}$ for predictors up to the $4^{\text {th }}$ order (red curve), whereas for higher orders no significant additional gain is obtained.

Let us now focus on the staggered SAR case. Fig. 7 shows the PRI sequence which has been employed for the present simulations. It consists of about 230 different PRIs, which are cyclically repeated during the staggered SAR acquisition (the dashed horizontal red line indicates the mean PRI, which is about $0.37 \mathrm{~ms})$. For the considered system, about $4 \%$ of the acquired raw data are affected by gaps [13]. Moreover, a 4-bit BAQ is up to now foreseen for the entire Tandem- $\mathrm{L}$ mission, which guarantees an interferometric coherence loss smaller than $1 \%$ [34], [35], hence minimizing the effects of quantization errors (the block size for BAQ is set to $N_{\text {Block }}=128$ range samples, which corresponds to a realistic block size implemented on board, e.g., the DLR satellites TerraSAR-X and TanDEM-X [34]). Based on the theoretic coding gains in Fig. 6 for a mean PRF of $2700 \mathrm{~Hz}$, a prediction order $N_{p} \leq 4$ is assumed in the following.

As performance measure, we have evaluated the signal-to-quantization noise ratio (SQNR) of the focused SAR image. The SQNR represents the figure of merit of a quantizer describing how much the output signal has been corrupted by

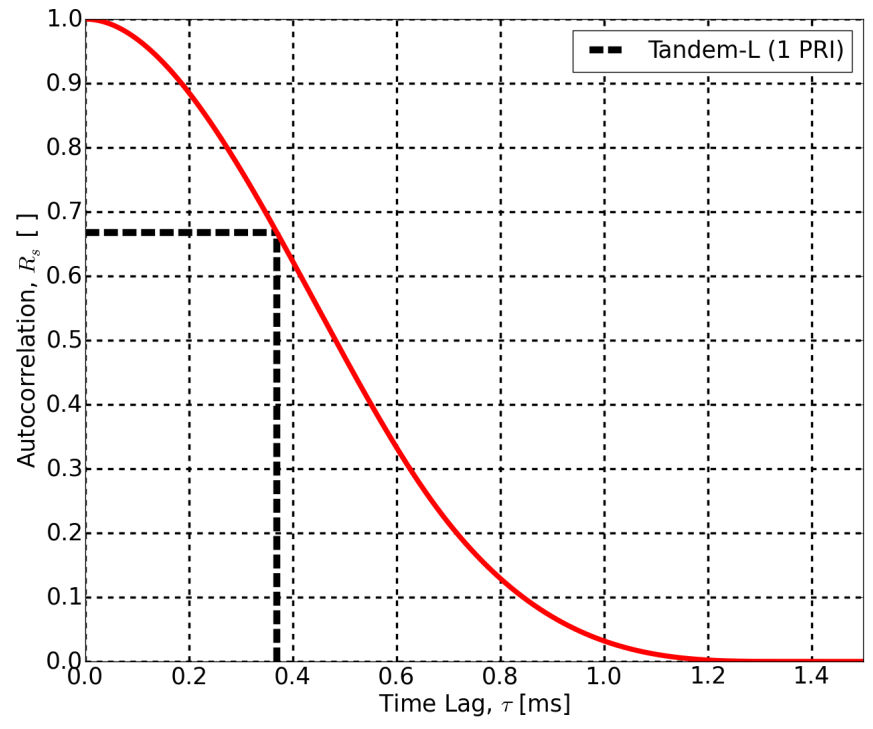

Fig. 5. Theoretical autocorrelation of the azimuth SAR raw data as a function of the mutual time shift $\tau$. For Tandem-L the mean PRI is about $0.37 \mathrm{~ms}$, which leads to a correlation of about 0.67 between adjacent azimuth samples (dashed black lines).

quantization noise. It is defined as the power ratio between the uncompressed SAR image $I$ and the quantization error $q=I-\hat{I}$ which affects the reconstructed image $\hat{I}$

$$
\text { SQNR }=\frac{\sum_{p=1}^{P}\left|I_{p}\right|^{2}}{\sum_{p=1}^{P}\left|q_{p}\right|^{2}},
$$

where $P$ represents the total number of pixels used for the estimation. The SQNR can be used for the evaluation of relevant interferometric SAR performance measures, such as the coherence loss due to quantization [36]

$$
\gamma_{\text {Quant }}=\frac{1}{1+\mathrm{SQNR}^{-1}} \text {. }
$$




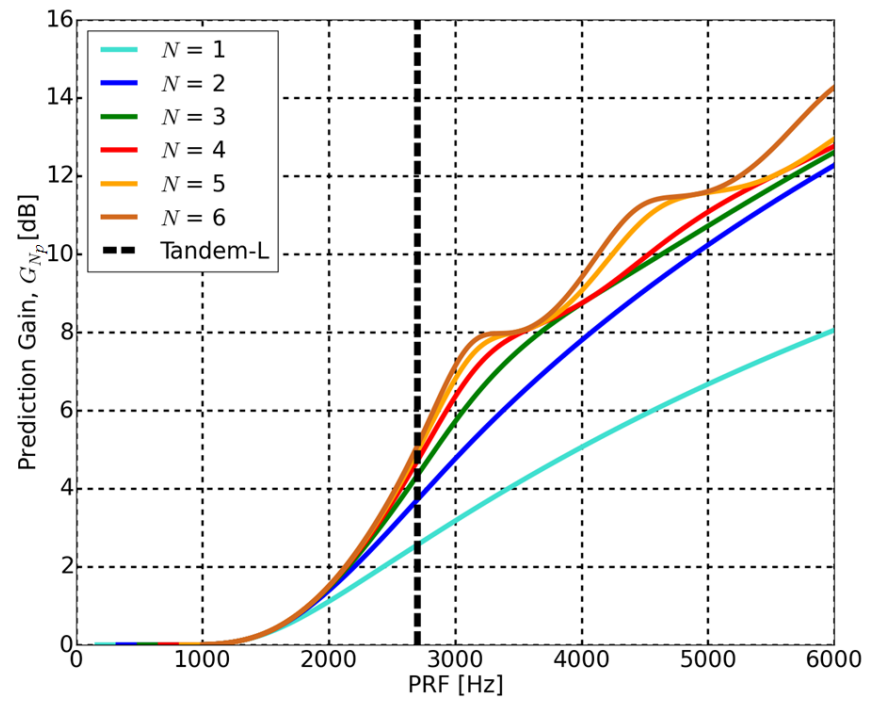

Fig. 6. Theoretical prediction gain $G_{N_{p}}$ for up to the $6^{\text {th }}$ order as a function of the PRF for a Tandem-L-like system (see Table II). The gains for a PRF of $2700 \mathrm{~Hz}$ are obtained in correspondence to the dashed black line.

The quantization coherence, in turn, is exploited to assess the resulting increase of interferometric phase errors, as discussed in [34], [36].

For this analysis, we generated non-uniformly sampled raw data and compressed them with the proposed DP-BAQ as well as with a standard BAQ for comparison; then, we applied a Best Linear Unbiased (BLU) interpolation to resample the non-uniform staggered SAR raw data on a uniform grid and, finally, SAR focusing was performed. By selecting the elaborated sequence of PRIs shown in Fig. 7, one can impose that no more than one sample at a time is missed in the azimuth direction [11]. Fig. 8 shows the SQNR obtained for a homogeneous target as a function of the average rate $\bar{R}$ and for different quantization schemes. The performance of a BAQ with constant bit rate is taken as reference and is given in black; then, the SQNR for the proposed DP-BAQ for different prediction orders is depicted as follows: $1^{\text {st }}$ order in turquoise, $2^{\text {nd }}$ order in blue, $3^{\text {rd }}$ order in green, and $4^{\text {th }}$ order in red. No significant additional gain is observed for prediction orders $N_{p}>4$. Assuming now as target performance the one obtained with a 4-bit BAQ, the proposed DP-BAQ allows for an improvement of SQNR of up to around $5.5 \mathrm{~dB}$. Alternatively, a $4^{\text {th }}$-order DP-BAQ at 3 bits/sample approximately provides the same SQNR of a 4-bit BAQ (both around $22 \mathrm{~dB}$, which corresponds to a negligible coherence loss of less than $1 \%$, i.e., $\gamma_{\text {Quant }}>0.99$ ), hence allowing for a data reduction of about $25 \%$. Analogously, if a 3-bit BAQ is used as reference, about 2.25 bits/sample can be used for the proposed method, corresponding again to a data reduction of about $25 \%$. When using lower compression rates, a poorer performance gain can be reasonably expected. This is due to the larger quantization noise affecting the raw data, which implies an additional loss in the azimuth correlation, hence resulting in a more "imprecise" prediction (i.e. a larger $\sigma_{d, N_{p}}^{2}$ in (12)). It is worth highlighting that the estimates of data rate reduction provided above are derived assuming that, for the reference

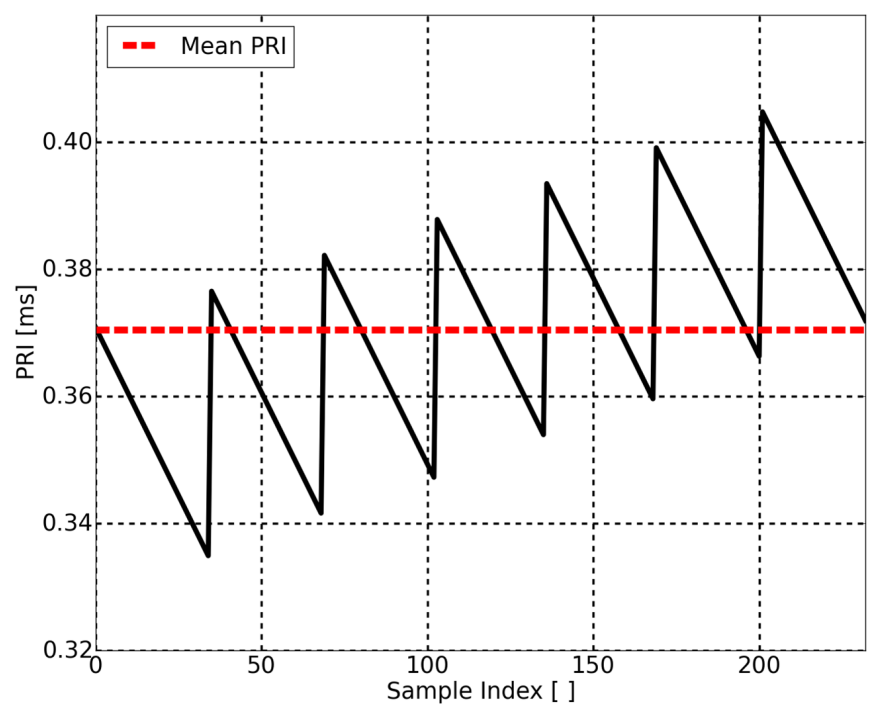

Fig. 7. Example of a PRI-sequence employed for the present simulations, which is cyclically repeated during a staggered SAR acquisition [13]. The dashed red line indicates the mean PRI, which is of about $0.37 \mathrm{~ms}$.

BAQ scenario (black curve), the missing raw data samples are treated as valid (gap-free) signals and are hence quantized with the corresponding number of bits (indicated on the x-axis of Fig. 8). If one assumes, on the other hand, that the missing samples can be actually "cut" from the raw data matrix and not downlinked at all, the effective data rate to be considered as reference reduces by about $4 \%$ (which corresponds to the percentage of acquired raw data affected by gaps for the present staggered SAR system). This implies a slightly lower data reduction of about $22 \%$ for both, the 3-bit and 4-bit case. If compared with the method proposed in [15], DP-BAQ results in general in a higher data rate, but allows, on the other hand, for a simpler and cheaper onboard implementation. Indeed, the prediction process basically consists of a linear combination of $N_{p} \leq 4$ range lines, while the data reduction technique in [15] typically requires the storage and processing of more than 15 range lines. Hence, the suggested algorithm can be performed in real time by using a single state-of-the-art FPGA, which has strong storage limitations making the onboard real-time implementation a cost-driving challenge. Moreover, the proposed method preserves the non-uniformly sampled SAR raw data that may be used for a more advanced on-ground processing as in [37] and [25].

Looking at Fig. 8, it can also be noticed that the SQNR values show, going from one integer rate to the next one, first a slow variation and then a steeper trend, which can be explained as follows: the fractional quantization rates shown in Fig. 8 are implemented by opportunely toggling the bit rate selection of an integer-bit BAQ quantizer along azimuth and/or range. This technique is named azimuth-switched quantization (ASQ) [18] and allows for higher flexibility of compression without increasing the overall system complexity. For this, let us assume a target non-integer bit rate $\bar{R}_{\text {frac }}$. According to [18], $\bar{R}_{\text {frac }}$ can be "synthesized" by means of a sequence of integer rates of length $N_{\text {seq }}$, where the next smaller integer rate $\left(\bar{R}_{\text {inf }}=\left\lfloor\bar{R}_{\text {frac }}\right\rfloor\right)$ occurs with a relative frequency $f \in[0,1]$, 


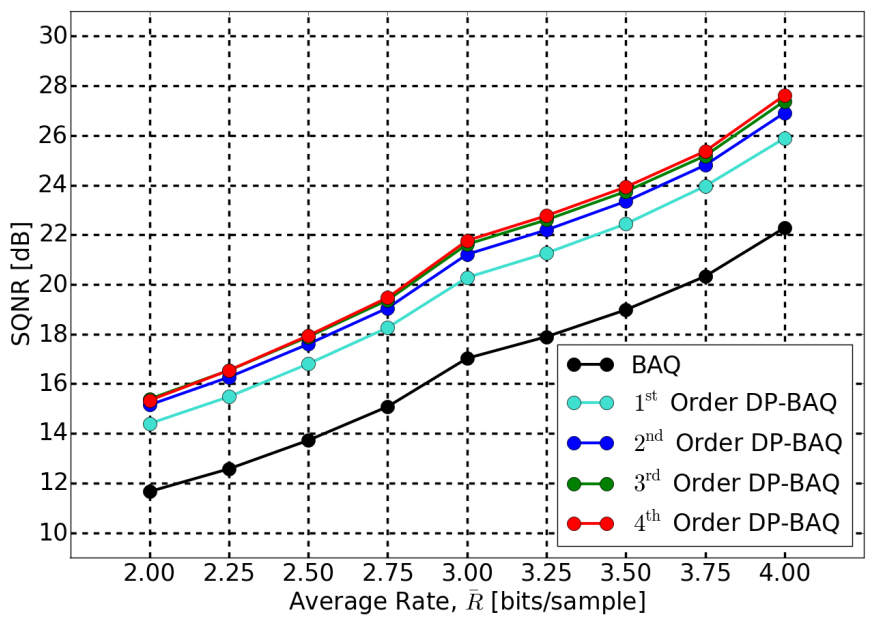

Fig. 8. Signal-to-quantization noise ratio (SQNR) obtained from a homogeneous SAR scene as a function of the average rate $\bar{R}$, for a standard BAQ with constant bitrate (black), and for the proposed DP-BAQ (up to the $4^{\text {th }}$ order), with variable bit rate allocation and dynamic selection of the prediction order in the gap vicinity.

and the next greater integer one $\left(\bar{R}_{\text {sup }}=\left\lceil\bar{R}_{\text {frac }}\right\rceil\right)$ occurs with a relative frequency $1-f$. By applying the described rate sequence of length $N_{\text {seq }}$ for the quantization of the SAR data, e.g. along azimuth, the expected $\mathrm{SQNR}_{\text {frac }}$ associated to the resulting non-integer rate is expressed as

$$
\mathrm{SQNR}_{\text {frac }}=\frac{\mathrm{SQNR}_{\text {sup }} \cdot \mathrm{SQNR}_{\text {inf }}}{f \cdot \mathrm{SQNR}_{\text {sup }}+(1-f) \cdot \mathrm{SQNR}_{\text {inf }}},
$$

being $\mathrm{SQNR}_{\text {inf }}$ and $\mathrm{SQNR}_{\text {sup }}$ the signal-to-quantization noise ratio associated to $\bar{R}_{\text {inf }}$ and $\bar{R}_{\text {sup }}$, respectively. As an example, the non-integer rate $\bar{R}=3.25$ bits/sample can be implemented by employing a sequence of, e.g., $N_{\text {seq }}=20$ bit rate values and selecting 15 times $\bar{R}_{\text {inf }}=3$ bits/sample (corresponding to a relative occurrence $f=0.75$ ) and the remaining 5 times $\bar{R}_{\text {sup }}=4$ bits/sample. The above equation is derived by simply weighting the noise power contributions associated to the integer rates according to the factor $f$. Moreover, it explains the non-linear trend shown by the SQNR for fractional bit rates and has been verified by the simulation results in Fig. 8. It is worth highlighting that possible variations in the image quality among neighboring pixels, resulting from the use of a variable bit rate along azimuth, are actually averaged after SAR processing, provided that the extension $L_{s e q}$ (in meters) of the azimuth sequence of length $N_{\text {seq }}$, used to synthesize the target fractional rate, is sufficiently smaller than the synthetic aperture $L_{s a}$, i.e., $L_{s e q}<<L_{s a}$ (indeed, $L_{s a}$ represents the azimuth distance within which the targets overlap their response in the raw data domain). These two quantities are expressed as

$$
\begin{gathered}
L_{s e q}=v_{s} \cdot \mathrm{PRI} \cdot N_{s e q}, \\
L_{s a}=\lambda \frac{R_{0}}{L},
\end{gathered}
$$

being $\lambda$ the radar wavelength (for $\mathrm{L}$ band, $\lambda=23.9 \mathrm{~cm}$ ) and $R_{0}$ the slant range distance. For the aforementioned example of $\bar{R}=3.25$ bits/sample, the corresponding sequence of length
$N_{\text {seq }}=20$ results in an azimuth extension of about 60 meters. In this case, $L_{s e q}$ is (more than) two orders of magnitude smaller than $L_{s a}$, which, for the above listed parameters, is in the order of a few tens of kilometers. As a consequence, the variable quality in the raw data is completely "smoothed" after data focusing and hence not appreciable in the resulting SAR and InSAR products, where, instead, a uniform performance loss is observed, as if an equivalent fractional bit rate $\bar{R}$ was used.

As it has been already pointed out, the main advantage of employing azimuth-switched quantization (ASQ) [18] is that it allows for a higher flexibility in terms of achievable compression rate and performance without increasing the onboard computational effort. Alternatively, native non-integer bit rates can be implemented by following a uniform quantizer with additional hardware/software blocks, such as an entropic (Huffman) coder. This solution achieves in general slightly better performance with respect to a traditional May-Lloyd non-uniform quantizer and constant length coding, such as BAQ [38], at the cost of an increased overall system complexity. However, as the length (i.e., the number of bits) of the encoded symbols is determined by the input signal statistics, the use of an entropic coder does not allow for exactly knowing in advance the volume of data required for a certain SAR acquisition (differently from ASQ, where indeed the data rate can be accurately calculated before the SAR survey), which causes additional complexity for the operation of SAR missions. A comparison between the two referred schemes is beyond the scope of this paper and will be subject of future research and investigations.

When considering the variable PRI shown in Fig. 7, for the application of predictive coding in staggered SAR systems one should in principle take into account the time-variant autocorrelation properties of the non-uniform azimuth SAR raw signal. However, we could verify that the performance of the predictor obtained by employing a set of weights derived for each one of the around 230 PRI intervals is practically the same as the one obtained by using, for all pulses, the weights derived from the mean PRI of about $0.37 \mathrm{~ms}$ (the difference in SQNR is less than $0.1 \mathrm{~dB}$ ). This means that, once the antenna pattern, the PRI sequence and the prediction order are defined, the resulting weights $\beta_{i}$ are constant values that can be derived before commanding, and then stored on board in registers and recalled by the predictor during the SAR data take.

The importance of exploiting the a-priori knowledge about the gap locations in order to dynamically adapt the order of the prediction filter is shown in Fig. 9, which depicts the SQNR estimated on the raw data for a homogeneous target as a function of the azimuth samples (the average over a large number of range samples is considered), and for different quantization schemes. In this example, two gaps are highlighted by the vertical grey lines (over each gap a null value is assumed). The performance of a standard BAQ is depicted in black and is obviously almost constant for all the samples, since the gaps do not have any impact on it. Then, the performance of a $4^{\text {th }}$-order prediction (P-BAQ) with fixed predictor order and constant bit rate at 4 bits/sample is shown in orange, and a clear drop of performance is visible after each 


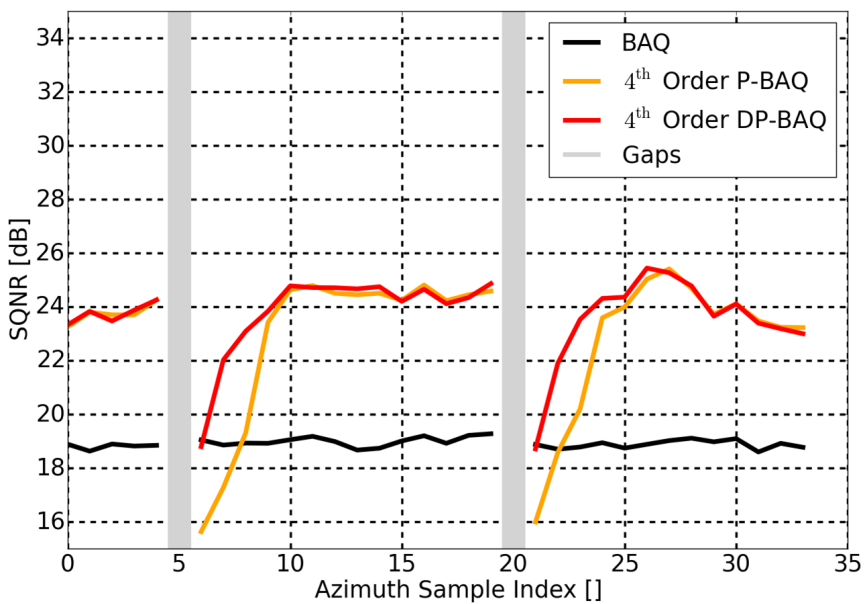

Fig. 9. Signal-to-quantization noise ratio (SQNR) as a function of the azimuth samples (averaged along range), for different quantization schemes: standard BAQ (black), predictive BAQ with constant bit rate and fixed $4^{\text {th }}$-order prediction (orange), and the proposed DP-BAQ with dynamic $4^{\text {th }}$-order prediction and constant bit rate (red). Two gaps are highlighted by the vertical grey lines, and a significant improvement in performance of 3-4 $\mathrm{dB}$ can be observed with the proposed method right after the gap occurrence, which outperforms all other considered approaches.

gap, as a consequence of the larger prediction error introduced by the missing sample. The prediction error gradually reduces (i.e. the SQNR increases) for the following samples, and the method reaches again its "regime" SQNR of about $24 \mathrm{~dB}$ after 5 samples, i.e. when the gap is not employed anymore by the prediction filter. A significant gain of about 3-4 $\mathrm{dB}$ is observed if a dynamic prediction order is selected after the gap occurrence (in red), according to the proposed DP-BAQ. It is worth noting that, in this example, we kept the bit rate constant in the gap vicinity, in order to better highlight the impact of the dynamic prediction order only on the final performance. Moreover, one can notice that the SQNR values observed in Fig. 9 are different, and in particular smaller, than those shown in Fig. 8. This is due to the fact that the latter are calculated on the focused SAR scene, where a processed Doppler bandwidth $\mathrm{PBW}=1130 \mathrm{~Hz}$ is applied, which is less than half of the PRF (being PRF $=2700 \mathrm{~Hz}$ ). Such a low-pass filtering operation contributes to mitigate the effect of digitization errors, since it averages out high-frequency contributions in the focused data and alleviates also the occurrence of saturation effects, ultimately resulting in a better quantization performance (on the other hand, for the raw data the full Doppler bandwidth is used).

In addition, we have evaluated the proposed method for the simulated SAR backscatter profile $\left(\sigma^{0}\right)$ depicted in Fig. 10 in brown, which shows a "jump" of $10 \mathrm{~dB}$ along the azimuth dimension. Such a large dynamic range typically occurs over highly inhomogeneous targets, such as, e.g., urban areas. The four graphs depict the SQNR as a function of the azimuth distance for different quantization schemes (nominal BAQ is depicted in black, $1^{\text {st }}$ order prediction in turquoise, $4^{\text {th }}$ order prediction in red) for a bit rate $\bar{R}=4$ bits/sample with the following settings applied in the gap vicinity: (a) fixed prediction order and constant bit rate; (b) fixed prediction
TABLE III

SQNR VALUES (IN DB) OBTAINED FOR ALL AVAILABLE COMPRESSION SCHEMES AND SETTING COMBINATIONS FOR THE SIMULATION SHOWN IN Fig. 10, MEASURED FOR AZIMUTH OF 20 KM, 40 KM AND 80 KM.

\begin{tabular}{|c|c|c|c|}
\hline Quant. scheme / Azimuth & 20 km & $40 \mathrm{~km}$ & $80 \mathrm{~km}$ \\
\hline$\overline{\text { BAQ }}$ & 22.2 & 20.4 & 21.6 \\
\hline BAQ variable $N_{b}$ & 23.3 & 21.5 & 22.4 \\
\hline $\mathbf{1}^{\text {st }}$-Order P-BAQ & 24.2 & 21.1 & 24.4 \\
\hline $\mathbf{1}^{\text {st }}$-Order P-BAQ variable $N_{b}$ & 25.6 & 22.4 & 25.9 \\
\hline $\mathbf{1}^{\text {st }}$-Order P-BAQ dynamic $N_{p}$ & 24.2 & 21.2 & 24.4 \\
\hline $1^{\text {st }}$-Order DP-BAQ & 25.6 & 22.4 & 25.9 \\
\hline $2^{\text {nd }}$-Order P-BAQ & 24.6 & 21.4 & 24.8 \\
\hline $2^{\text {nd }}$-Order P-BAQ variable $N_{b}$ & 26.6 & 22.8 & 26.7 \\
\hline $2^{\text {nd }}$-Order P-BAQ dynamic $N_{p}$ & 25.0 & 21.6 & 25.2 \\
\hline $2^{\text {nd }}$-Order DP-BAQ & 26.6 & 22.9 & 26.9 \\
\hline $3^{\text {rd }}$-Order P-BAQ & 24.4 & 21.3 & 24.3 \\
\hline $\mathbf{3}^{\text {rd }}$-Order P-BAQ variable $N_{b}$ & 26.7 & 22.8 & 26.0 \\
\hline $3^{\text {rd }}$-Order P-BAQ dynamic $N_{p}$ & 25.4 & 21.8 & 25.6 \\
\hline $3^{\text {rd }}$-Order DP-BAQ & 27.1 & 23.1 & 27.4 \\
\hline $4^{\text {th }}$-Order P-BAQ & 24.1 & 21.0 & 23.6 \\
\hline $4^{\text {th }}$-Order P-BAQ variable $N_{b}$ & 26.5 & 22.6 & 25.1 \\
\hline $4^{\text {th }}$-Order P-BAQ dynamic $N_{p}$ & 25.5 & 21.9 & 25.8 \\
\hline $4^{\text {th }}$-Order DP-BAQ & 27.4 & 23.2 & 27.6 \\
\hline
\end{tabular}

order and variable bit rate (the latter employed also for BAQ, see also Table I); (c) dynamic prediction order and constant bit rate; (d) DP-BAQ with dynamic prediction order and variable bit rate (the latter employed also for BAQ). Again, if a fixed prediction order is employed (Fig. 10 (a)), the presence of gaps (for the considered staggered SAR system, about $4 \%$ of the acquired raw data are affected by gaps) degrades the reconstruction so much that a $4^{\text {th }}$-order predictor performs worse than a $1^{\text {st }}$-order one. On the other hand, the use of a dynamic prediction order (as for the DP-BAQ in Fig. 10 (d)), together with a distributed bit rate allocation in the gap neighborhood, significantly improves the resulting performance, which can be exploited to reduce the resulting data rate. The increase in performance due to the optimized bit rate allocation around the gap (hence, disregarding the gain introduced by the predictive coding) can be noticed when comparing, e.g., Fig. 10 (a) with Fig. 10 (b) (or, equivalently, Fig. 10 (c) with Fig. 10 (d)) and is in the range between $1 \mathrm{~dB}$ and $2 \mathrm{~dB}$ for all compression schemes. Moreover, it can be noticed that, for the $1^{\text {st }}$-order prediction (turquoise line) the performance gain due to the employment of a dynamic prediction order is practically negligible. Indeed, for $N_{p}=1$ the prediction is less affected by the presence of a gap (due to the shorter memory of the prediction filter). On the other hand, the gain in SQNR becomes significant for larger values of $N_{p}$, as shown by the red line $\left(N_{p}=4\right)$. In order to provide a quantitative estimation of the resulting performance, Table III shows the values of SQNR obtained from the profiles in Fig. 10 for all setting combinations of the considered quantization schemes (the performance for a $2^{\text {nd }}$-order and a $3^{\text {rd }}$-order predictor is reported as well for completeness), for an azimuth of $20 \mathrm{~km}, 40 \mathrm{~km}$, and $60 \mathrm{~km}$. From this, the resulting SQNR gains can be better appreciated, and the corresponding coherence loss $\gamma_{\text {Quant }}$ ranges between $0.2 \%$ (for the maximum 


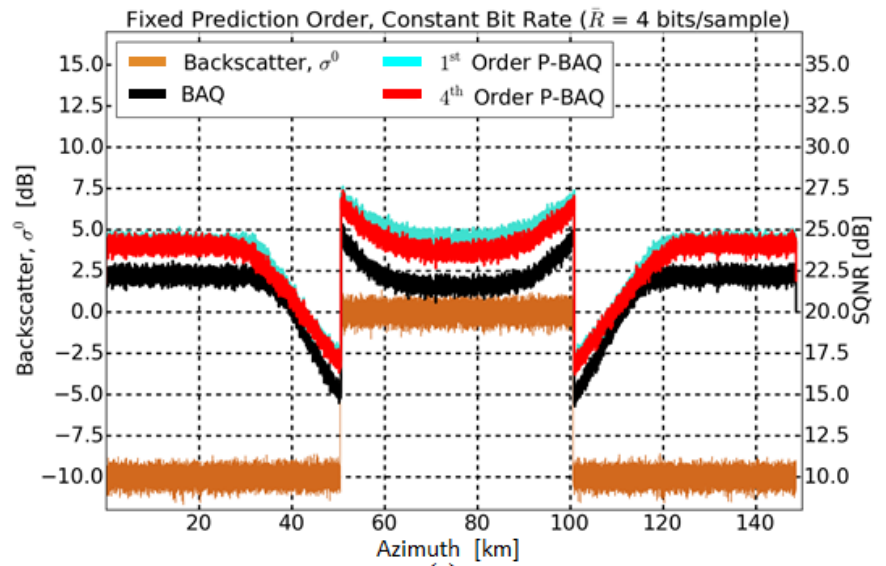

(a)

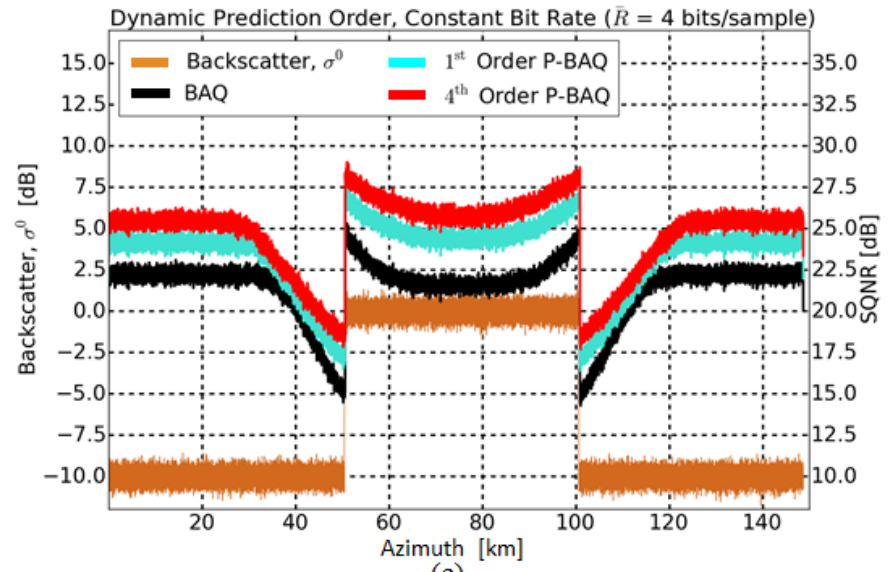

(c)

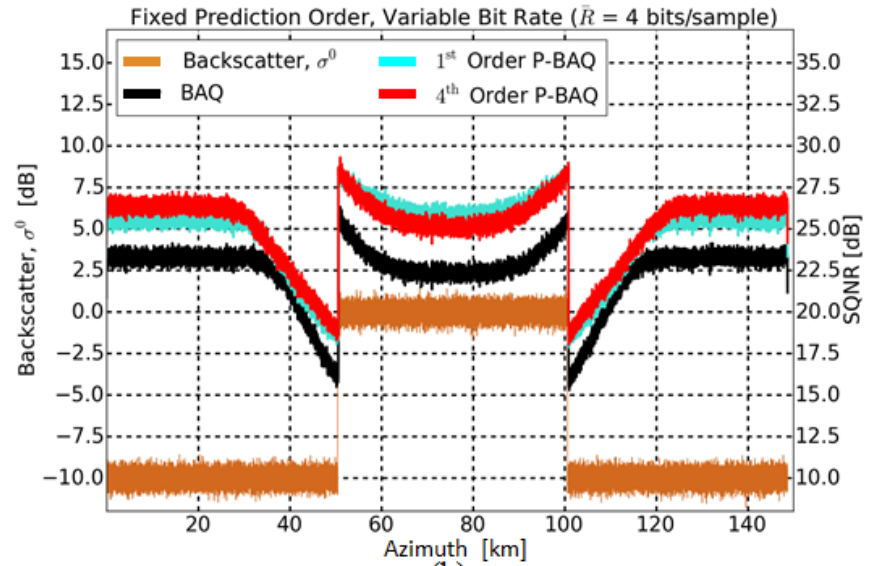

(b)

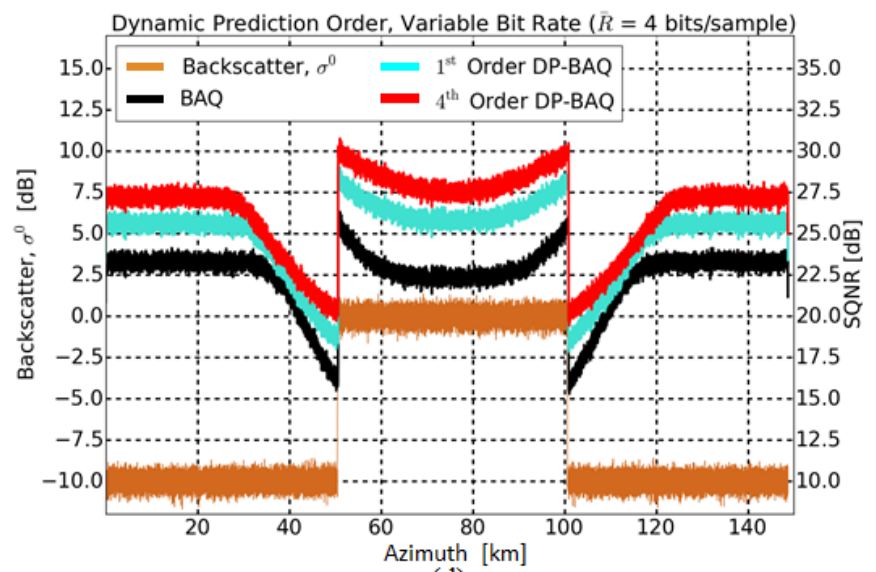

(d)

Fig. 10. Backscatter profile $\left(\sigma^{0}\right.$, in brown) and corresponding SQNR as a function of the azimuth distance for different quantization schemes (BAQ in black, $1^{\text {st }}$ order prediction in turquoise, $4^{\text {th }}$ order prediction in red) with the following settings applied in the gap vicinity: (a) fixed prediction order and constant bit rate; (b) fixed prediction order and variable bit rate (the latter employed also for BAQ); (c) dynamic prediction order and constant bit rate; (d) DP-BAQ with dynamic prediction order and variable bit rate (the latter employed also for BAQ). The curves are derived for an average rate $\bar{R}$ of 4 bits/sample. The use of a dynamic prediction order, together with an optimized bit rate allocation in the gap neighborhood, significantly improves the resulting performance, which can be exploited to reduce the resulting data rate.

SQNR of $27.6 \mathrm{~dB}$ ) and about $1 \%$ (for the minimum SQNR of $20.4 \mathrm{~dB})$.

A significant loss in SQNR is observed for all considered compression techniques over the area of low backscatter (with a mean backscatter of $-10 \mathrm{~dB}$ ) close to the high-backscatter one. Such a performance degradation is due to the masking effect caused by the presence of high-backscatter targets in close vicinity and "propagates" up to a distance comparable with the synthetic aperture $L_{s a}$ [34], [39]. Indeed, $L_{s a}$ is expressed in (24) and, for the considered system $(\lambda=23.9 \mathrm{~cm}$, $L=10 \mathrm{~m}$, and, for an elevation angle $\theta_{e}=40^{\circ}$ considered in this simulation, $R_{0}$ is in the order of $900 \mathrm{~km}$ ), it results that $L_{s a} \approx 22 \mathrm{~km}$. Such a distance approximately corresponds to the extension up to which the SQNR profiles are affected to the left of the first discontinuity, at $50 \mathrm{~km}$ azimuth (which extends from about $30 \mathrm{~km}$ to $50 \mathrm{~km}$ ), and to the right of the second discontinuity, at $100 \mathrm{~km}$ azimuth (which extends from about $100 \mathrm{~km}$ to $120 \mathrm{~km}$ ). The described effect strongly affects the reconstruction in the focused SAR image: as an example, in Fig. 10 (a) the SQNR varies from 15-17.5 dB in the low-backscatter area (and $\gamma_{\text {Quant }} \approx 3 \%$ ) up to about
$25-27.5 \mathrm{~dB}$ in the high-backscatter one (and $\gamma_{\text {Quant }}<0.5 \%$ ).

\section{CONCLUSION}

In this paper, onboard data volume reduction in staggered SAR systems is addressed. Such systems require the acquisition of a large volume of data for the imaging of wide swath widths with fine azimuth resolution. Staggered SAR raw data samples exhibit a certain correlation in azimuth, which is introduced by the antenna pattern and a significant oversampling. The proposed method is based on the use of linear predictive coding, which aims at removing the data redundancy by means of an efficient encoding and quantization of the azimuth SAR raw samples. In particular, for the present investigations a causal predictor has been considered, i.e., only preceding samples are used in the prediction process. Dynamic Predictive Block-Adaptive Quantization (DP-BAQ) exploits the a-priori knowledge of the position of the gaps occurring during the staggered SAR operation, by adaptively selecting the bit rate and the prediction order in the gap vicinity, in order to improve the resulting performance. We conducted simulations for a Tandem-L-like L-band staggered 
SAR system for different compression settings (in terms of quantization scheme, prediction order, and bit rate allocation strategy) and SAR backscatter distributions, showing that predictive quantization allows for a significant reduction of the onboard data volume by requiring, at the same time, a modest processing effort for its onboard implementation. The proposed technique will be applied and verified on real staggered SAR data, similar to [14], as object of further research and publications. Furthermore, the investigation of alternative prediction techniques, such as non-causal and/or non-linear prediction schemes, or the inclusion of polar quantization methods, will be a topic for possible studies and could be considered for the design of future SAR systems, where the combined use of even larger PRFs and/or oversampling factors will lead to a further improvement of the data reduction capacity.

\section{ACKNOWLEDGMENT}

The authors gratefully acknowledge the valuable suggestions of the anonymous reviewers. This work has received funding from the European Unions Horizon 2020 research and innovation programme under grant agreement No. 822014 (www.s4pro-h2020.eu).

\section{REFERENCES}

[1] A. Currie and M. A. Brown, "Wide-swath SAR," vol. 139, no. 2, pp. 122-135, 1992

[2] G. D. Callaghan and I. D. Longstaff, "Wide swath spaceborne SAR using a quad element array," vol. 146, no. 3, pp. 159-165, 1999.

[3] M. Suess, B. Grafmüller, and R. Zahn, "A novel high resolution, wideswath SAR system," in Proc. IGARSS, Sydney, Australia, July 2001, pp. 1013-1015.

[4] M. Younis, C. Fischer, and W. Wiesbeck, "Digital beamforming in SAR systems," IEEE Trans. on Geosci. and Remote Sens., vol. 41, no. 7, pp. 1735-1739, July 2003.

[5] G. Krieger, N. Gebert, and A. Moreira, "Unambiguous SAR signal reconstruction from non-uniform displaced phase center sampling," IEEE Geosci. and Remote Sens. Lett., vol. 1, no. 4, pp. 260-264, October 2004.

[6] N. Gebert, G. Krieger, and A. Moreira, "Digital beamforming on receive: Techniques and optimization strategies for high-resolution wide-swath SAR imaging," IEEE Trans. Aerosp. Electron. Syst., vol. 45, no. 2, pp. 564-592, April 2009.

[7] G. Krieger, N. Gebert, M. Younis, and A. Moreira, "Advanced synthetic aperture radar based on digital beamforming and waveform diversity," in Proc. IEEE Radar Conf., Rome, Italy, May 2008, pp. 767-772.

[8] A. Freeman, G. Krieger, P. Rosen, M. Younis, W. Johnson, S. Huber, R. Jordan, and A. Moreira, "SweepSAR: Beam-forming on receive using a reflector-phased array feed combination for spaceborne SAR," in Proc. IEEE Radar Conf., Pasadena, CA, USA, May 2009, pp. 1-9.

[9] B. Grafmüller and C. Schaefer, "High-resolution synthetic aperture radar device and antenna for one such radar," US Patent US 8013778B2, 12 22, 2005.

[10] G. Krieger, N. Gebert, M. Younis, F. Bordoni, A. Patyuchenko, and A. Moreira, "Advanced concepts for ultra-wide-swath SAR imaging," in Proc. EUSAR, Friedrichshafen, Germany, June 2008, pp. 1-4.

[11] M. Villano, G. Krieger, and A. Moreira, "Staggered SAR: High-resolution wide-swath imaging by continuous PRI variation," IEEE Trans. Geosci. and Remote Sens., vol. 52, no. 7, pp. 4462-4479, July 2014.

[12] _ "A novel processing strategy for staggered SAR," IEEE Geosci. Remote Sens. Lett., vol. 11, no. 11, pp. 1891-1895, November 2014.

[13] M. Villano, "Staggered synthetic aperture radar," Ph.D. dissertation, Karlsruhe Institute of Technology, Wessling, Germany, 2016, DLR-Forschungsbericht 2016-16, ISSN 1434-8454.
[14] M. Villano, G. Krieger, M. Jäger, and A. Moreira, "Staggered SAR: Performance analysis and experiments with real data," IEEE Trans. on Geosci. and Remote Sens., vol. 55, no. 11, pp. 6617-6638, November 2017.

[15] M. Villano, G. Krieger, and A. Moreira, "Onboard processing for data volume reduction in high-resolution wide-swath SAR," IEEE Geosci. Remote Sens. Lett., vol. 13, no. 8, pp. 1173-1177, August 2016.

[16] D. Lancashire, B. Barnes, and S. Udall, "Block adaptive quantization," US Patent US 6255987, 07 3, 2001

[17] R. Kwok and W. T. K. Johnson, "Block adaptive quantization of Magellan SAR data," IEEE Trans. on Geosci. and Remote Sens., vol. 27, no. 4, pp. 375-383, July 1989.

[18] M. Martone, B. Bräutigam, and G. Krieger, "Azimuth-Switched Quantization for SAR systems and performance analysis on TanDEM-X data," IEEE Geosci. Remote Sens. Lett., vol. 11, no. 1, pp. 181-185, January 2014

[19] T. Algra, "Data compression for operational SAR missions using entropy-constrained block adaptive quantization," in Proc. Int. Geosci. Remote Sens. Symp., Toronto, Canada, June 2002, pp. 1135-1138.

[20] E. Attema, C. Cafforio, M. Gottwald, P. Guccione, A. M. Guarnieri, F. Rocca, and P. Snoeij, "Flexible dynamic block adaptive quantization for Sentinel-1 SAR missions," IEEE Geosci. Remote Sens. Lett., vol. 7 , no. 4, pp. 766-770, October 2010.

[21] P. Guccione and A. M. Guarnieri, "A space adaptive quantizer for spaceborne SAR," IEEE Trans. Geosci. Remote Sens., vol. 49, no. 10, pp. 3564-3573, October 2011.

[22] P. Guccione, M. Scagliola, and D. Giudici, "Principal components dynamic block quantization for multichannel SAR," in IEEE Int. Geosci. Remote Sens. Symp., Bejing, China, July 2016, pp. 2090-2093.

[23] M. Martone, M. Villano, M. Younis, and G. Krieger, "Efficient Onboard Quantization for Multi-Channel SAR Systems," IEEE Geosci. Remote Sens. Lett., Accepted for publication, 2019.

[24] N. Jayant and P. Noll, Digital Coding of Waveforms-Principles and Applications to Speech and Video. New Jersey: Prentice-Hall, 1984.

[25] M. Pinheiro, P. Prats-Iraola, M. Rodriguez-Cassola, and M. Villano, "Combining spectral estimation and BLU interpolation for the reconstruction of low-oversampled staggered SAR data," in Proc. EUSAR, Aachen, Germany, June 2018, pp. 1-6.

[26] E. Magli and G. Olmo, "Lossy predictive coding of SAR raw data," IEEE Trans. Geosci. and Remote Sens., vol. 41, no. 5, pp. 977-987, May 2003.

[27] T. Ikuma, M. Naraghi-Pour, and T. Lewis, "Predictive quantization of range-focused SAR raw data," IEEE Trans. Geosci. and Remote Sens., vol. 50, no. 4, pp. 1340-1348, April 2012.

[28] I. H. McLeod, I. G. Cumming, and M. S. Seymour, "ENVISAT ASAR data reduction: impact on SAR interferometry," IEEE Trans. Geosci. Remote Sens., vol. 36, no. 2, pp. 589-602, March 1998.

[29] G. Schreier, SAR geocoding: data and systems. Wichmann, 1993.

[30] S. Barbarossa, "Detection and imaging of moving objects with synthetic aperture radar. Part 1. Optimal detection and parameter estimation theory." IEE Proceedings F - Radar Signal Processing, vol. 139, no. 1, pp. 79-88, January 1992.

[31] A. Gersho and R. M. Gray, Vector quantization and signal compression. Springer Science \& Business Media, 2012.

[32] R. M. Narayanan, D. W. Doerr, and D. C. Rundquist, "Temporal decorrelation of X-band backscatter from wind-influenced vegetation,' IEEE Trans. Aerosp. Electron. Syst., vol. 28, no. 2, pp. 404-412, April 1992.

[33] A. Moreirma, G. Krieger, M. Younis, I. Hajnsek, K. Papathanassiou, M. Eineder, and F. De Zan, "Tandem-L: A mission proposal for monitoring dynamic earth processes," in Proc. Int. Geosci. Remote Sens. Symp., Vancouver, Canada, July 2011, pp. 1385-1388.

[34] M. Martone, B. Bräutigam, and G. Krieger, "Quantization effects in TanDEM-X data," IEEE Trans. Geosci. and Remote Sens., vol. 53, no. 2, pp. 583-597, 2015.

[35] M. Martone, B. Bräutigam, P. Rizzoli, C. Gonzalez, M. Bachmann, and G. Krieger, "Coherence evaluation of TanDEM-X interferometric data," ISPRS J. of Photogr. Remote Sens., vol. 73, pp. 21-29, September 2012.

[36] R. Bamler and P. Hartl, "Synthetic Aperture Radar Interferometry," Inverse Problems, vol. 14, no. 4, pp. 1-54, Feb. 1998.

[37] G. Krieger, S. Huber, M. Villano, M. Younis, T. Rommel, P. Lopez-Dekker, F. Q. de Almeida, and A. Moreira, "CEBRAS: Cross elevation beam range ambiguity suppression for high-resolution wide-swath and MIMO-SAR imaging," in Proc. Int. Geosci. Remote Sens. Symp., Milan, Italy, July 2015, pp. 196-199.

[38] A. Gersho, "Principles of quantization," IEEE Trans. Circuits Syst., vol. 25, no. 7, pp. 427-436, July 1978. 
[39] S. Huber, M. Younis, and G. Krieger, "The TanDEM-X mission: overview and interferometric performance," Int. J. Microw. Wireless Technol., vol. 2, no. 3-4, pp. 379-389, July 2010. 